\title{
Pre- and postnatal nutrition in sheep affects $\beta$-cell secretion and hypothalamic control
}

\author{
Anna H Kongsted, Sanne V Husted, Malin P Thygesen', Vibeke G Christensen, \\ Dominique Blache $^{2}$, Anders Tolver ${ }^{3, *}$, Torben Larsen ${ }^{4}$, Bjørn Quistorff ${ }^{5}$ \\ and Mette $O$ Nielsen
}

Department of Veterinary Clinical and Animal Sciences, Faculty of Health and Medical Sciences, University of Copenhagen, Copenhagen, Denmark

${ }^{1}$ Cook Medical Europe APS, Bjaeverskov, Denmark ${ }^{2}$ School of Animal Biology, University of Western Australia, Perth, Western Australia, Australia

${ }^{3}$ Department of Basic Sciences and Environment, Faculty of Health and Medical Sciences, University of Copenhagen Copenhagen, Denmark

${ }^{4}$ Department of Animal Science, Faculty of Science and Technology, Aarhus University, Aarhus, Denmark

${ }^{5}$ Department of Biomedical Sciences, Faculty of Health and Medical Sciences, Nuclear Magnetic Resonance Centre, University of Copenhagen, Copenhagen, Denmark

*(A Tolver is now at Department of Mathematical Sciences, Faculty of Science, University of Copenhagen,

Copenhagen, Denmark)

\author{
Correspondence \\ should be addressed \\ to A H Kongsted \\ Email \\ ahtp@sund.ku.dk
}

\begin{abstract}
Maternal undernutrition increases the risk of type 2 diabetes and metabolic syndrome later in life, particularly upon postnatal exposure to a high-energy diet. However, dysfunctions of, for example, the glucose-insulin axis are not readily detectable by conventional tests early in life, making it difficult to identify individuals at risk. Thus, other methods are required. We hypothesised that prenatally undernourished individuals (but not postnatally overnourished ones) are adapted to a life with limited food availability, which would be evident under conditions reflecting starvation, stress and short-term abundance of food. In this study, twinpregnant sheep were fed diets meeting $100 \%$ (NORM) or $50 \%$ (LOW) of energy and protein requirements during the last trimester. Twin offspring were fed either a normal moderate (CONV) diet or a high-carbohydrate-high-fat (HCHF) diet from 3 days to 6 months of age (approximately puberty) and the same moderate diet thereafter until 2 years of age (young adulthood; only females), resulting in four groups: NORM-CONV, LOW-CONV, NORM-HCHF and LOW-HCHF. At the age of 6 months and 2 years respectively, they were subjected to fasting and propionate (nutrient abundance) and adrenalin challenges. At 6 months of age, postnatal HCHF diet exposure caused metabolic alterations, reflecting hypertriglyceridaemia and altered pancreatic $\beta$-cell secretion. Irrespective of postnatal diet, prenatal undernutrition was found to be associated with unexpected endocrine responses of leptin, IGF1 and cortisol during fasting (lack of or the opposite response compared with the controls) in 2-year-old adults. In conclusion, a HCHF diet interfered with $\beta$-cell function, whereas maternal undernutrition did not lead to any changes in the LOW offspring, except to abnormal hormone responses, suggesting that fetal programming interferes with hypothalamic integration of important endocrine axis.
\end{abstract}

\section{Key Words}

- prenatal programming

- obesity

- fasting

- propionate tolerance test

- gluconeogenesis

- glucose

- insulin

- IGF1

- leptin

- cortisol
Journal of Endocrinology (2013) 219, 159-171 http://joe.endocrinology-journals.org DOI: 10.1530/JOE-13-0099
C 2013 Society for Endocrinology Printed in Great Britain
Published by Bioscientifica Ltd 


\section{Introduction}

The prevalence of metabolic syndrome and type 2 diabetes (T2D) is increasing in an epidemic-like fashion, and it is particularly pronounced in developing countries currently undergoing rapid economic transition and experiencing increased wealth gain (Misra \& Vikram 2004, Spalding et al. 2009). Studies in humans and animals have shown that low birth weight and in utero growth restriction can predispose to developing obesity and metabolic disorders later in life, which according to the so-called predictive adaptive hypothesis can be explained by the programming/adaptation of the metabolic and endocrine functions of the foetus to make it prepared to be born into a world with stressful conditions and scarcity of food (Gluckman et al. 2005). However, the metabolic disorders may only become manifested if the individual is exposed to mismatching nutritional conditions after birth (Vickers et al. 2000, Cleal et al. 2007, Fall et al. 2008), which the programming is not adapted to. The very early postnatal life, and even the first few weeks in humans, may be very important in this respect, and small changes in diets and growth patterns during the first few weeks and months after birth may have long-term impacts on the individual and increase the risk of developing obesity (Forsen et al. 2000, Stettler et al. 2003, 2005). This points to the importance of identifying as early in life as possible individuals who have undergone 'programming' or whose in utero environment and early postnatal environments are mismatched and thus are at a risk of adverse health outcomes later in life. The conventional tests to study glucose-insulin axis function are not adequate in this respect, as the negative consequences of fetal programming detectable by these tests most often do not become evident until the individuals approach adulthood (Petry et al. 2001, Gavete et al. 2005).

Therefore, in this study, we aimed to study whether the impacts of late-gestation undernutrition on metabolic and endocrine plasticity can be assessed relatively early in life (adolescence) by exposure to challenges reflecting stressful conditions (adrenalin tolerance test) with scarcity of feed (fasting) requiring hepatic gluconeogenesis (propionate tolerance test). Thus, these tests could be reflective of early programming at a time when conventional glucose tolerance tests cannot be used to identify individuals at a risk of developing metabolic diseases. Second, we investigated how an energy-dense high-fat diet would affect later disease outcomes. We used the Copenhagen sheep model, which has been described previously (Nielsen et al. 2012). The sheep model was chosen for this study due to the emphasis on late-gestation impacts, where sheep and humans have similar developmental patterns in the last trimester: their offspring are born physiologically precocial, which means that they are much more mature at birth than rodent and porcine offspring, which are born altricial. We have reported previously that prenatal undernutrition in late gestation can programme for reduced insulin sensitivity in postnatal life. Feeding a mismatching obesogenic high-fat diet in early postnatal life also had dramatic effects on glucose-insulin axis function (reduced insulin-secretory ability of $\beta$-cells and pancreatic fibrosis), but only as long as the diet was fed, and upon diet correction and normalisation of body fat, these traits were no longer evident in adult sheep (Kongsted et al. 2013). Therefore, we further hypothesised that the time of birth is a critical set point for permanent programming outcomes and hence needed an animal model where this issue could be addressed.

\section{Materials and methods}

\section{Experimental animals and design}

The experimental design and the Copenhagen model have been described previously (Nielsen et al. 2012). All experimental animal handling and procedures were approved by The Danish National Committee on Animal Experimentation. Two prenatal and two postnatal nutritional treatments were applied in a $2 \times 2$ factorial design. Twenty-one Shropshire twin-pregnant sheep (ovis) were assigned to one of the two feeding treatments during the last 6 weeks of gestation (term $=147$ days): a diet meeting $100 \%$ (NORM, $n=10$ ) or $50 \%$ (LOW, $n=11$ ) of the daily requirements for energy and protein for a normal twinpregnancy. Twin lambs were separated from their dams and fed either of the postnatal diets from 3 days to 6 months (around puberty) of age: one twin lamb was fed a moderate hay (CONV, $n=21)$ diet supplemented with milk replacer during the first 8 weeks and the diet was adjusted weekly to achieve moderate weight gains ( $225 \mathrm{~g} /$ day), and the other was fed a high-carbohydratehigh-fat (HCHF, $n=21$ ) diet consisting of high-fat dairy cream (38\% fat; ad libitum until the maximal daily allowance of $0.5 \mathrm{l} /$ day was reached), high-starch popped maize (ad libitum until the maximal daily allowance of $1.0 \mathrm{~kg} /$ day was reached) and milk replacer (ad libitum until the maximal daily allowances were reached, which were $2.01 /$ day until 8 weeks of age and $0.51 /$ day thereafter).

Published by Bioscientifica Ltd. 
Thus, each twin from a twin pair was always fed a different diet and was allocated to one of the postnatal groups CONV or HCHF in a way to have comparable groups with respect to the range of birth weights and gender. This generated four experimental groups: NORM-CONV $(n=10)$, NORM-HCHF $(n=9)$, LOW-CONV $(n=10)$, and LOW-HCHF $(n=9)$. All animals were subjected to a range of metabolic and endocrine challenge tests (described below) at 6 months of age, and later half the animals (all males and three females) were killed. The remaining offspring (only females) were fed the same moderate haybased diet from 6 months to 2 years of age (young adulthood), and they were again subjected to metabolic and endocrine challenge tests and then killed.

\section{Fasting challenge test in 6-month-old lambs and 2-year-old adult sheep}

To determine whether metabolic adaptations to limited food supplies could be detected during starvation in programmed animals, we subjected all the lambs to a 2-day-long fast.

Catheters were inserted into both jugular veins at least 1 day prior to the fasting challenge as described previously (Husted et al. 2007).

For 6-month-old adolescent lambs, food supply was stopped in the afternoon at $1530 \mathrm{~h}($ time $=0)$, and the lambs were subsequently fasted for $47 \mathrm{~h}$. At $44 \mathrm{~h}$, the lambs were subjected to a propionate tolerance test, as described below. Blood samples were collected at hours 0 , $16,24,40,44$ (prior to propionate injection), 45, 46 (1 and $2 \mathrm{~h}$ after propionate injection), and 48 ( $1 \mathrm{~h}$ after re-feeding). The HCHF-diet fed animals were only given maize at re-feeding to eliminate interference of very high plasma triglyceride (TG) levels in hormone analyses.

For the 2-year-old adult female sheep, the fasting period was extended to $71 \mathrm{~h}$ to provide a metabolic load similar to that provided to the smaller adolescents, and blood samples were collected at hours $0,16,24,40,48,64$ and 72 ( $1 \mathrm{~h}$ after re-feeding).

\section{Propionate tolerance test in 6-month-old fasted adolescent lambs}

To detect the gluconeogenic capacity of the liver and how the individual experimental group handled an overload of nutrient supply, a propionate challenge was applied.

Propionate $\left(2.5 \mathrm{ml} / \mathrm{kg}\right.$ body weight ${ }^{0.75}$ of a $2.5 \mathrm{M}$ propionic acid solution (the pharmacy, Faculty of Life
Sciences, University of Copenhagen, Denmark)) was injected intravenously after $44 \mathrm{~h}$ of fasting (time $=0$ in the propionate tolerance test). Blood samples were collected $-5,5,10,15,30$ and $60 \mathrm{~min}$ after injection. The propionate challenge was omitted in the adult sheep.

\section{Adrenalin tolerance test in 6-month-old adolescent lambs and 2-year-old adult sheep}

During short-term stress exposure, the experimental groups may react differently depending on their programmed metabolism. Therefore, we subjected the adolescent lambs and adult sheep to an adrenalin tolerance test $2 \mathrm{~h}$ after feeding with a normal morning meal (only maize and not cream at 6 months of age). Adrenalin $\left(0.25 \mathrm{~g} / \mathrm{kg}\right.$ body weight ${ }^{0.75}$; DAK, Nycomed, Roskilde, DK) diluted in $\mathrm{NaCl}$ solution was injected intravenously at time $=0$, and blood samples were collected $-5,2.5,10,20,30$ and 60 min after injection.

\section{Processing and biochemical measurements in blood samples}

Blood samples were collected in EDTA and heparin vials and kept chilled until centrifugation at $1800 \boldsymbol{g}_{\text {av }}$ for 15 min at $4{ }^{\circ} \mathrm{C}$, and thereafter plasma was separated and stored at $-20^{\circ} \mathrm{C}$ until analysis.

The concentrations of glucose, non-esterified fatty acids (NEFAs), triacylglycerides (TAGs), urea, creatinine, lactate, $\beta$-hydroxybutyrate (BOHB) and $\gamma$-glutamyltransferase (GGT) were measured in plasma samples obtained at all the sampling points during fasting and propionate tolerance test, whereas the concentrations of the hormones insulin, growth hormone (GH), insulin-like growth factor 1 (IGF1), cortisol and leptin were measured only in samples obtained at 0,44 and $48 \mathrm{~h}$ from the 6-month-old fasted adolescent lambs (except that of cortisol due to lack of a sample) and in those obtained at $0,24,48,64$ and $72 \mathrm{~h}$ from the 2-year-old adult fasted sheep. Samples obtained after the adrenalin tolerance test were analysed for glucose and NEFA concentrations.

Plasma glucose, TG, urea, lactate, creatinine and GGT concentrations were determined according to standard procedures (Siemens Diagnostics Clinical Methods for ADVIA 1650) using an autoanalyser, ADVIA 1650 Chemistry System (Siemens Medical Solutions, Tarrytown, NY, USA).

Plasma NEFA, BOHB, insulin, IGF1, GH and leptin concentrations were measured as described previously (Kiani et al. 2011). Plasma cortisol concentration was

Published by Bioscientifica Ltd. 
determined using a commercial RIA kit (Clinical Assays, GammaCoat, Cortisol 125I RIA Kit, DiaSorin, Søborg, Denmark). The samples were assayed in duplicate in $25 \mu \mathrm{l}$ aliquots and run in one assay. The intra-assay coefficient of variation for samples containing 41.0 and $89.5 \mathrm{nmol}$ cortisol/1 was 5.2 and $2.7 \%$ respectively.

\section{Statistical analyses}

At each sampling time point, a two-way ANOVA was used to test for differences in the plasma levels of metabolites or insulin between the treatment groups. Repeated measurements over time obtained during fasting and during propionate and adrenalin tolerance tests were analysed using a mixed-effects model for repeated measurements including fixed effects of preand postnatal treatments and sampling time. To study the response in a tolerance test, all the statistical analyses were carried out on variables adjusted for baseline levels measured just before the test. All variables, except plasma glucose concentrations, were log-transformed before the statistical analyses. For glucose and insulin measurements during the propionate tolerance test, the area under the curve (AUC) from time points 0-15 and 15-120 min was computed as AUC glucose and AUC $_{\text {insulin. }}$ We decided to split AUC into two parts separated by the time of the peak level, and separate two-way ANOVAs were carried out on the two measures to test for differences between the treatment groups. A $P$ value $<0.05$ was considered statistically significant. All analyses were carried out using R: A language and environment for statistical computing ( $\mathrm{R}$ Development Core Team 2009).

\section{Results}

It should be noted that at 6 months of age, both female and male lambs were studied, but at 2 years of age, only the females were studied. Therefore, gender effects could be evaluated from the data obtained from the 6-month-old lambs. On the other hand, effects related to diet alteration could be evaluated based on data obtained from the females, which were studied at both 6 months and 2 years of age. Results for animal performance (e.g. maternal characteristics and weights, birth weights and growth patterns) and glucose-insulin sensitivity have been reported elsewhere (Nielsen et al. 2012, Kongsted et al. 2013). There were no significant effects of either gender or age, unless specifically stated in the following.

\section{Plasma metabolite and endocrine responses during 2 days of fasting in 6-month-old adolescent lambs}

Metabolites Glucose There was no difference in prefasting glucose concentrations between the groups, and glucose concentrations decreased in all the lambs in a similar manner ( $P=0.95$, Fig. 1A). After $47 \mathrm{~h}$ of fasting and $3 \mathrm{~h}$ after a propionate bolus injection (see below), the animals were re-fed (time $=180 \mathrm{~min}$, Fig. 1A). One hour after re-feeding, glucose concentrations increased significantly in the HCHF lambs but not in the CONV lambs (Fig. 1A).

Lactate The HCHF lambs had lower pre-fasting lactate concentrations compared with the CONV lambs (Fig. 1B). Lactate concentrations generally increased over the first $24 \mathrm{~h}$ of fasting in all the groups, except in the LOW-CONV group. Lactate concentrations decreased thereafter $(P<0.05)$ to the lowest levels after $44 \mathrm{~h}$ of fasting, with the exception of those in the LOW-CONV group, in which they remained at pre-injection levels at this time point. The HCHF females exhibited greater increases in lactate concentrations when corrected for baseline levels when compared with the males $(P<0.001)$.

Non-esterified fatty acids The HCHF lambs had the highest $(\sim 700 \mu \mathrm{m})$ pre-fasting NEFA concentrations (Fig. 1C). During fasting, NEFA concentrations increased in all the lambs $(P<0.001)$, but they were generally highest in the HCHF lambs, although the CONV lambs exhibited relatively greater NEFA responses than the HCHF lambs (as marked by the crosses in Fig. 1C). Upon re-feeding, NEFA concentrations decreased, most markedly in the HCHF lambs (Fig. 1C, marked with crosses), but to similarly low levels in all the groups.

Triglycerides Prior to fasting, the HCHF lambs had TG concentrations almost three times as high as those present in the CONV lambs (Fig. 1D). Even though TG concentrations decreased in the HCHF lambs during fasting, the HCHF lambs continued to exhibit the highest TG concentrations during fasting. One hour after re-feeding, TG concentrations decreased markedly in only the HCHF lambs (marked with crosses in Fig. 1D).

вонв Pre-fasting BOHB concentrations were significantly lower in the NORM-HCHF groups than in all the other groups (Fig. 1E). During fasting, BOHB concentrations increased significantly in the HCHF lambs than in the CONV lambs and also increased significantly in the prenatally undernourished LOW lambs than in the NORM

Published by Bioscientifica Ltd 
A

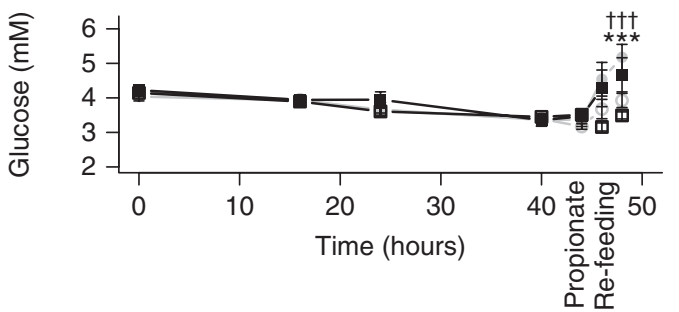

C

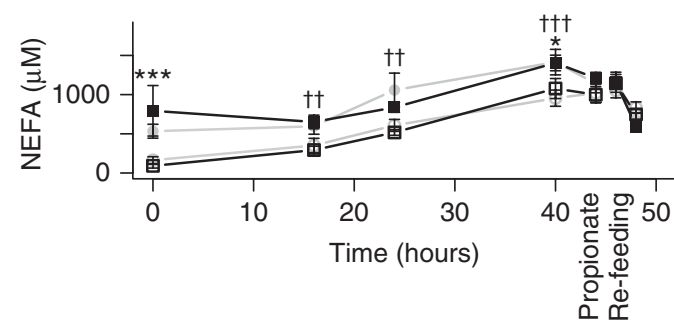

$\mathrm{E}$

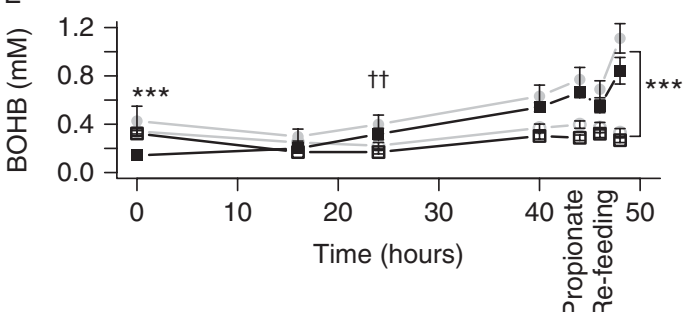

G

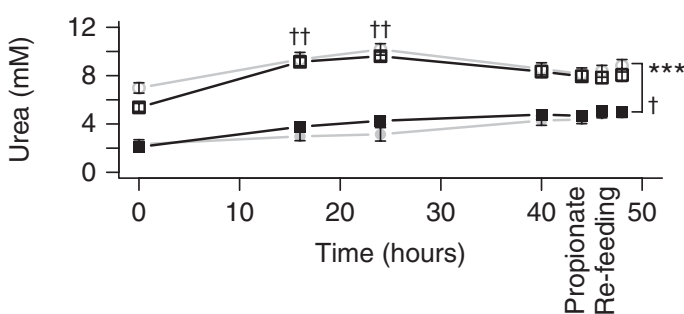

Figure 1

Metabolic responses during fasting. Lamb offspring (6 months) previously exposed to either a normal (NORM) diet or $50 \%$ undernutrition (LOW) during late gestation and either a conventional (CONV) or a highcarbohydrate-high-fat (HCHF) diet in postnatal life were subjected to 2 days of fasting, starting at time $=0 \mathrm{~h}$. After $44 \mathrm{~h}$ of fasting, a bolus of propionate $\left(2.5 \mathrm{ml} / \mathrm{kg}^{0.75}\right.$ of a $2.5 \mathrm{M}$ solution) was injected into the jugular vein. They were all re-fed at time $=47 \mathrm{~h}$. (A) Glucose, (B) lactate, (C) NEFA, (D) triglyceride, (E) $\beta$-hydroxybutyrate (BOHB), (F) $\gamma$-glutamyltransferase

lambs $(P<0.01)$. Upon re-feeding, BOHB concentrations increased even further in the HCHF lambs, but not in the CONV lambs (Fig. 1E).

$\gamma$-Glutamyltransferase The HCHF lambs had markedly higher plasma GGT concentrations than the CONV lambs prior to and during fasting (Fig. 1F).

Urea Prior to and during fasting, urea concentrations in the HCHF lambs were lower than those in the CONV
B

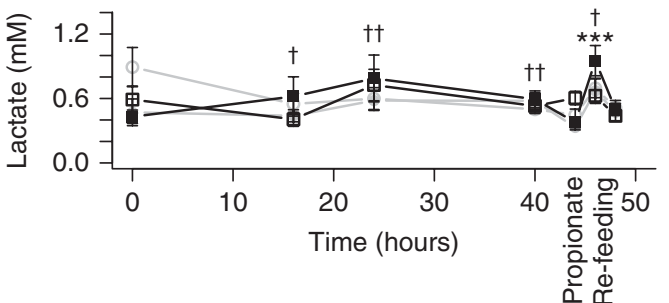

$\mathrm{D}$

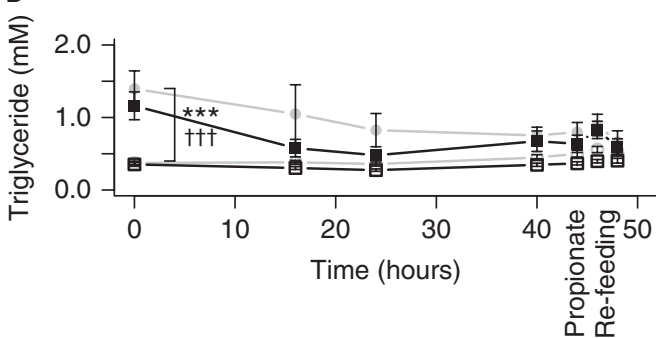

F
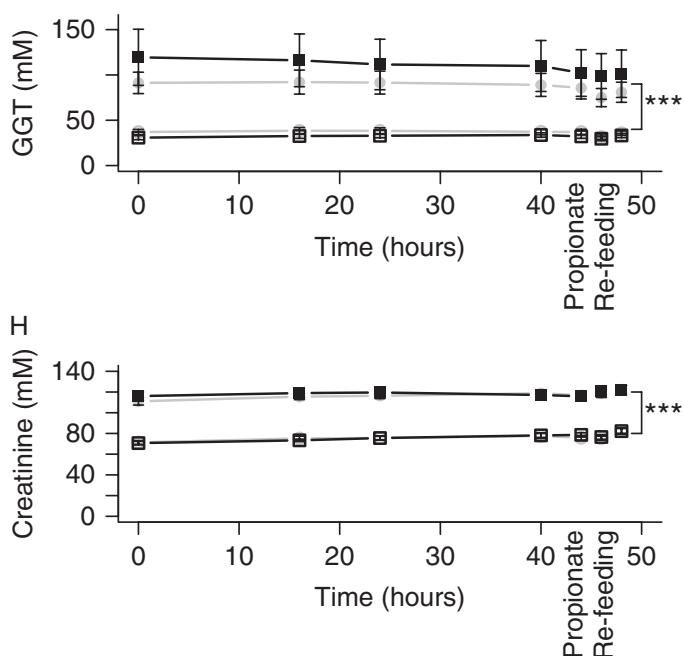

(GGT), (G) urea and (H) creatinine concentrations. White box: NORM-CONV (males $n=2$ and females $n=7$ ), black box: NORM-HCHF (males $n=5$ and females $n=4$ ), white circle: LOW-CONV (males $n=5$ and females $n=4$ ), and black circle: LOW-HCHF (males $n=5$ and females $n=5$ ); $* /{ }^{\dagger} P<0.05$, $* * /{ }^{\dagger \dagger} P<0.01$, and $* * * /{ }^{t+\dagger} P<0.001$, where $*$ denotes a difference in the actual concentration and the response corrected for the baseline value. The NORM offspring are shown in black and the LOW offspring in grey.

lambs (Fig. 1G). Urea concentrations increased during fasting in all the lambs $(P<0.001)$, but the increase was particularly pronounced in the HCHF lambs over the first $24 \mathrm{~h}$.

Creatinine Creatinine concentrations were highest in the HCHF lambs (Fig. 1H), i.e. the reverse of what was observed for urea, and remained relatively constant throughout the fasting period in all the treatment groups. 
Hormones Insulin There were no differences among the groups with regard to insulin concentrations prior to or during fasting (Fig. 2A), and they decreased in a comparable way in all the lambs. After re-feeding, they increased in only the HCHF lambs but not in the CONV lambs (Fig. 2A).

IGF1 and GH The HCHF lambs had higher pre-fasting IGF1 concentrations than the NORM lambs (Fig. 2B), and males had higher concentrations than the females $(P<0.05$; data not shown). During the $44 \mathrm{~h}$ of fasting, IGF1 concentrations decreased to comparable levels in all the lambs, and re-feeding did not alter this pattern (Fig. 2B). There were no significant differences in $\mathrm{GH}$ concentrations prior to $(P=0.09$, data not shown) or during $(P=0.91$, data not shown) fasting.
Leptin Leptin concentrations were significantly higher in the HCHF lambs than in the CONV lambs prior to fasting (Fig. 2C). Over the $44 \mathrm{~h}$ of fasting, leptin concentrations decreased to about half the pre-fasting levels, but prefasting group differences were maintained. Re-feeding increased plasma leptin concentrations in all the groups $(P<0.001$, Fig. 2C), except in the LOW-CONV group, which did not respond to re-feeding.

\section{Propionate induced changes in plasma metabolite and hormone concentrations in 6-month-old adolescent lambs after a 44-h fasting period}

Glucose Marked increases in plasma glucose concentrations were observed in all the treatment groups after a
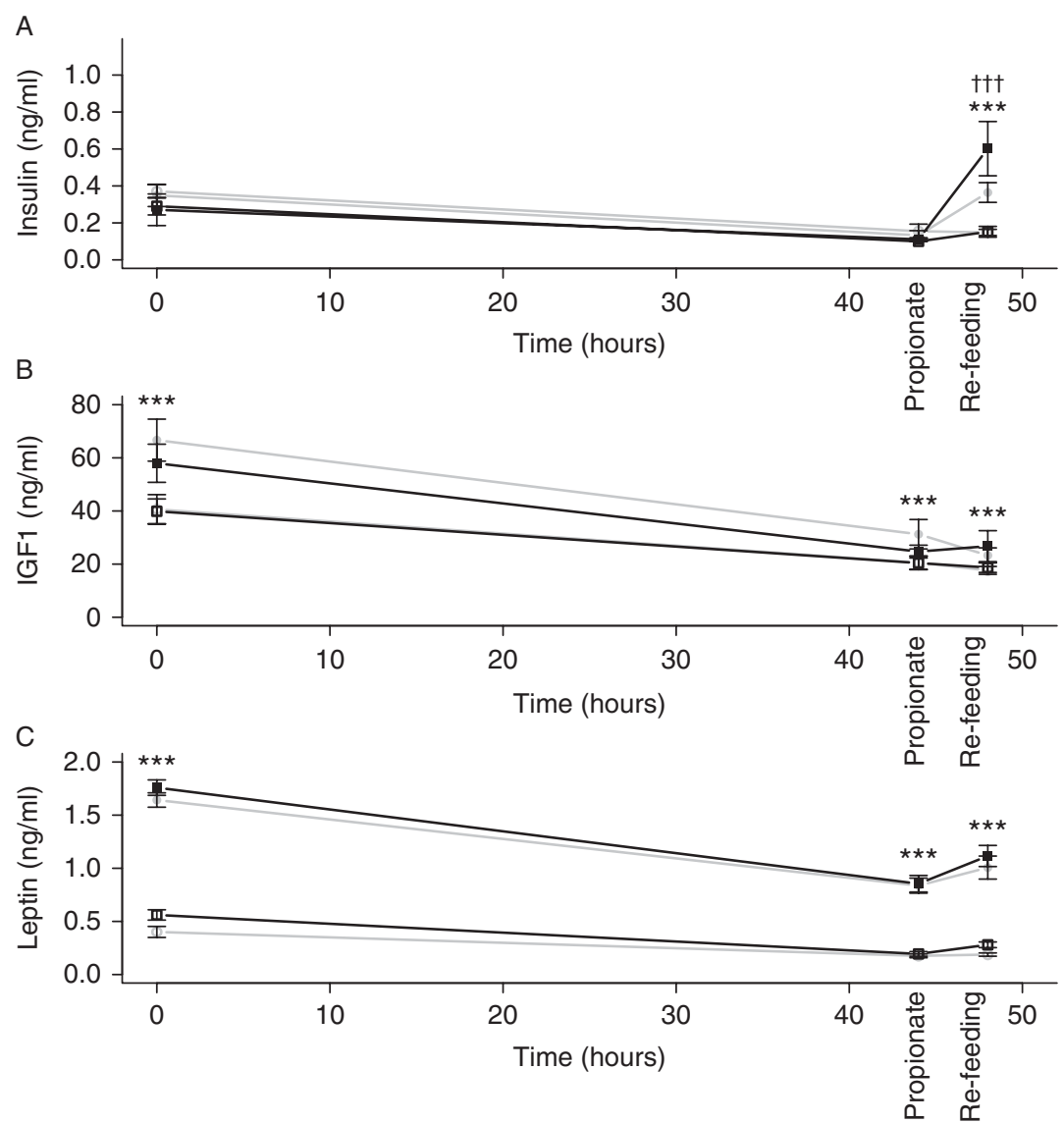

Figure 2

Hormone responses during fasting. Lamb offspring (6 months) previously exposed to either a normal (NORM) diet or $50 \%$ undernutrition (LOW) during late gestation and either a conventional (CONV) or a highcarbohydrate-high-fat (HCHF) diet in postnatal life were subjected to 2 days of fasting, starting at time $=0 \mathrm{~h}$. They were all re-fed at time $=47 \mathrm{~h}$. (A) Insulin, (B) IGF1 and (C) leptin concentrations. White box: NORM-CONV (males $n=2$ and females $n=7$ ), black box: NORM-HCHF (males $n=5$ and females $n=4$ ), white circle: LOW-CONV (males $n=5$ and females $n=4$ ), and black circle: LOW-HCHF (males $n=5$ and females $n=5$ ); $* /^{\dagger} P<0.05$, $* * /{ }^{\dagger+} P<0.01$, and $* * * /{ }^{t+\dagger} P<0.001$, where $*$ denotes a difference in the actual concentration and $t$ the response corrected for the baseline value. The NORM offspring are shown in black and the LOW offspring in grey. 
bolus injection of the gluconeogenic precursor propionate (Fig. 3A). After maximum glucose concentrations had been achieved, the HCHF lambs cleared the glucose less efficiently, and their plasma glucose concentrations remained higher 60 and 120 min after injection compared with those in the CONV lambs. Only in the NORM-CONV lambs did the glucose concentrations return to baseline levels within $1 \mathrm{~h}$ of injection.

Insulin All the lambs responded to propionate by increased insulin secretion, but peak responses, as for glucose, were observed earlier in the CONV lambs (10 min) than in the HCHF lambs (15 min) (Fig. 3B).
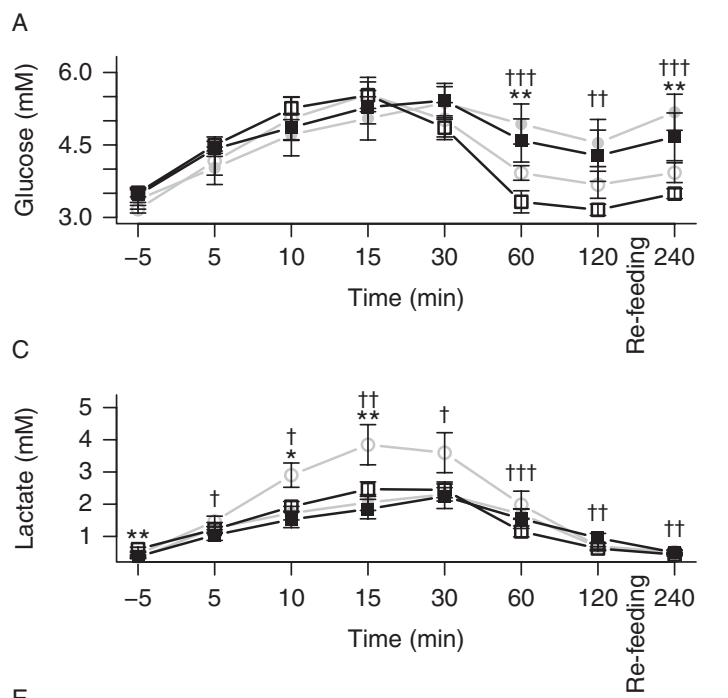

$E$
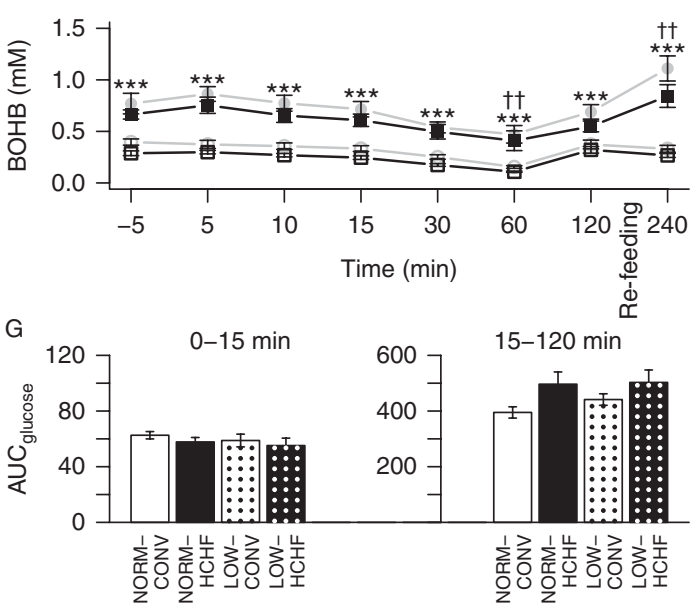

Figure 3

Metabolite and hormone responses during a propionate challenge test in the fasted lambs. The lambs were subjected to $44 \mathrm{~h}$ of fasting, starting at time $=0 \mathrm{~h}$. After $44 \mathrm{~h}$ of fasting, a bolus of propionate $\left(2.5 \mathrm{ml} / \mathrm{kg}^{0.75}\right.$ of a $2.5 \mathrm{M}$ solution) was injected into the jugular vein. They were all re-fed at time $=71 \mathrm{~h}$. (A) Glucose, (B) insulin, (C) lactate, (D) NEFA, (E) BOHB and (F) triglyceride concentrations and $(\mathrm{G}) A \cup C_{\text {glucose }}$ and $(H) A U C_{\text {insulin. }}$.
The magnitude of the insulin response in the HCHF lambs was significantly lower than that in the CONV adolescents 5, 10, 15 and $30 \mathrm{~min}$ after propionate injection (Fig. 3B). Two hours (time $=120 \mathrm{~min}$; Fig. 3B) after propionate injection, the concentrations returned to pre-injection levels in all the groups. However, after re-feeding, a slight increase was observed in the HCHF lambs.

Area under the curve $A_{U C}$ glucose was significantly higher in the HCHF lambs than in the CONV lambs at the time point $15-120 \mathrm{~min}(P<0.05$, Fig. 3G). $\mathrm{AUC}_{\text {insulin }}$ was lowest in the HCHF lambs during the first 15 min after propionate injection $(P<0.001$, Fig. $1 \mathrm{H})$.

B
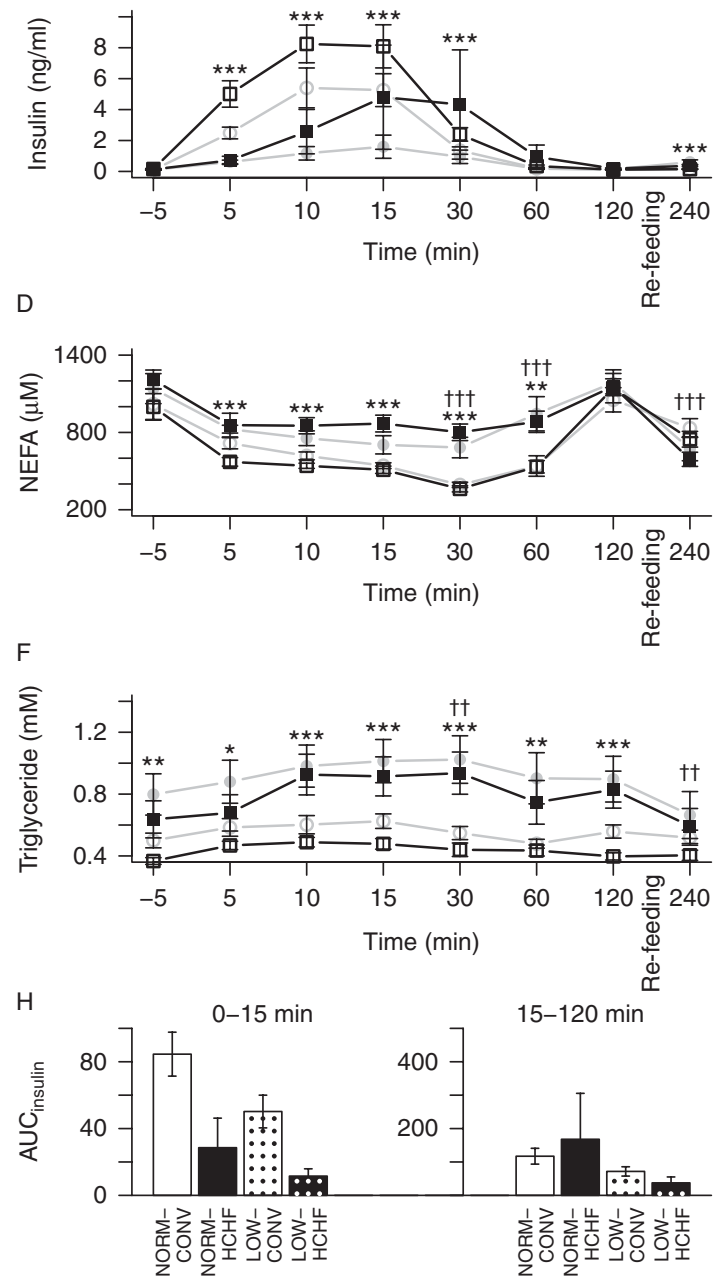

White box: NORM-CONV (females $n=4$ ), black box: NORM-HCHF (females $n=4$ ), white circle: LOW-CONV (females $n=5$ ), and black circle: LOW-HCHF (females $n=4$ ); $* I^{\dagger} P<0.05, * * /{ }^{\dagger+} P<0.01$, and $* * * /{ }^{+\dagger} P<0.001$ where * denotes a difference in the actual concentration and t the response corrected for the baseline value. The NORM offspring are shown in black and the LOW offspring in grey.

Published by Bioscientifica Ltd. 
The AUC $\mathrm{Clucose}_{\text {: }} \mathrm{AUC}_{\text {insulin }}$ ratio was highest in the HCHF lambs during both the period before peak glucose values had been obtained $(0-15 \mathrm{~min} ; P<0.001)$ and the period after peak values had been obtained (15-120 min; $P=0.01$ ) (data not shown).

Lactate Pre-injection plasma lactate concentrations in the 44-h-fasted lambs were highest in the LOW-CONV group (Fig. 3C), but similar in the other groups. Lactate concentrations increased markedly in all the groups within the first 15 (CONV) to 30 (HCHF) min of propionate injection, but the increase was highest in the LOW lambs and particularly in the LOW-CONV lambs (Fig. 3C). Within $120 \mathrm{~min}$ of injection, lactate concentrations returned to pre-injection levels.

It is worth noting that lactate followed the same pattern of change as glucose both during fasting and during the propionate challenge test, except in the LOW-CONV lambs, which had much more pronounced lactate responses (Fig. 3A and C).

Non-esterified fatty acids NEFA concentrations decreased in all the groups $(P<0.05$, Fig. 3D), particularly in the CONV lambs, and remained so until $30 \mathrm{~min}$ after injection, and later they returned to pre-injection levels, i.e. a pattern opposite to that observed for glucose and insulin. The HCHF lambs generally had the highest NEFA concentrations and smallest quantitative changes in plasma levels (Fig. 3D).

BOHB Pre-injection plasma BOHB concentrations after $44 \mathrm{~h}$ of fasting were higher in the HCHF lambs than in the CONV lambs (Fig. 3E). After propionate injection, BOHB concentrations decreased within the next $60 \mathrm{~min}$ of injection, but treatment differences persisted.

Triglycerides Pre-injection plasma TG concentrations were highest in the HCHF lambs (Fig. 3F). TG concentrations decreased over the first $60 \mathrm{~min}$ after the propionate injection in all the groups and thereafter they increased.

\section{Plasma glucose and NEFA responses in 6-month-old adolescent lambs and 2-year-old adult sheep during adrenalin tolerance test in the fed state}

Lambs There were no differences in glucose concentrations prior to adrenalin injection between the treatment groups (Fig. 4A). Plasma glucose concentrations increased in all the groups after adrenalin injection and were most pronounced in the HCHF lambs.
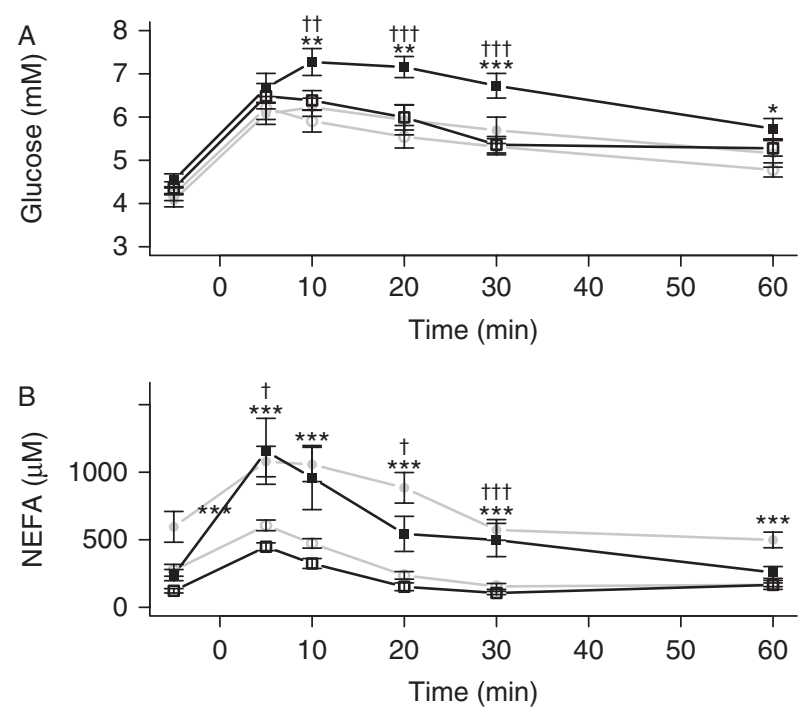

Figure 4

Adrenalin challenge. Adrenalin $\left(0.25 \mathrm{~g} / \mathrm{kg}\right.$ body weight $\left.{ }^{0.75}\right)$ was injected into the jugular vein. (A) Glucose and (B) NEFA concentrations. White box: NORM-CONV (females $n=4$ ), black box: NORM-HCHF (females $n=4$ ), white circle: LOW-CONV (females $n=5$ ), and black circle: LOW-HCHF (females

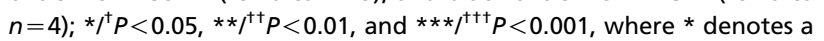
difference in the actual concentration and $t$ the response corrected for the baseline value. The NORM offspring are shown in black and the LOW offspring in grey.

Pre-injection NEFA concentrations in the fed state were highest in lambs exposed to prenatal LOW nutrition $(P=0.008)$ or postnatal HCHF nutrition ( $P=0.01$, Fig. $4 \mathrm{~B})$. After adrenalin injection, NEFA concentrations increased when compared with baseline levels in all the groups, remaining highest in the HCHF adolescents $(P<0.001)$.

Adult sheep Irrespective of the pre- or postnatal diet, after 1.5 years on a control diet, there was no difference in NEFA and glucose concentrations in the 2-year-old females (data not shown).

\section{Fasting induced changes in plasma metabolite and hormone concentrations in 2-year-old adult female sheep}

There were no differences in glucose, lactate, TG, BOHB, NEFA, GH (data for metabolites not shown) or insulin concentrations (Fig. 5A) between the groups either prior to or during fasting. On the other hand, a clear effect of the prenatal dietary exposure became evident in the adult sheep with respect to both baseline levels of leptin and cortisol and patterns of changes for leptin, IGF1 and cortisol during fasting (Fig. 5B, C, and D). This was not observed earlier in the same sheep at 6 months of age. In the LOW sheep, plasma IGF1 concentrations increased unexpectedly compared with baseline levels during

Published by Bioscientifica Ltd 
A

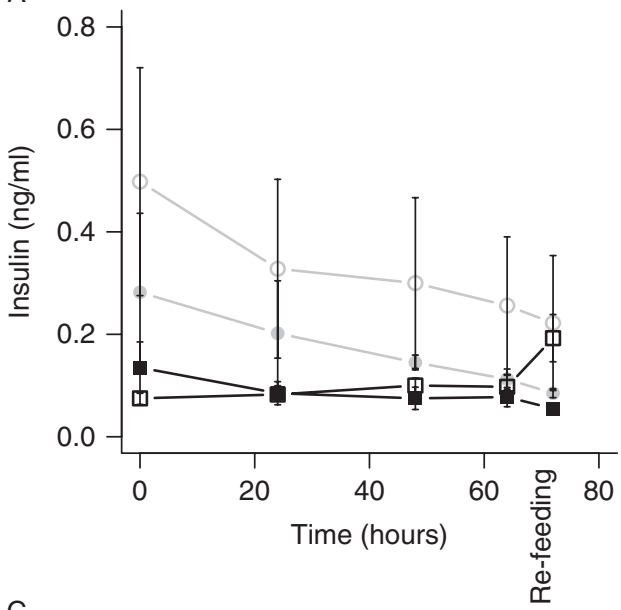

C

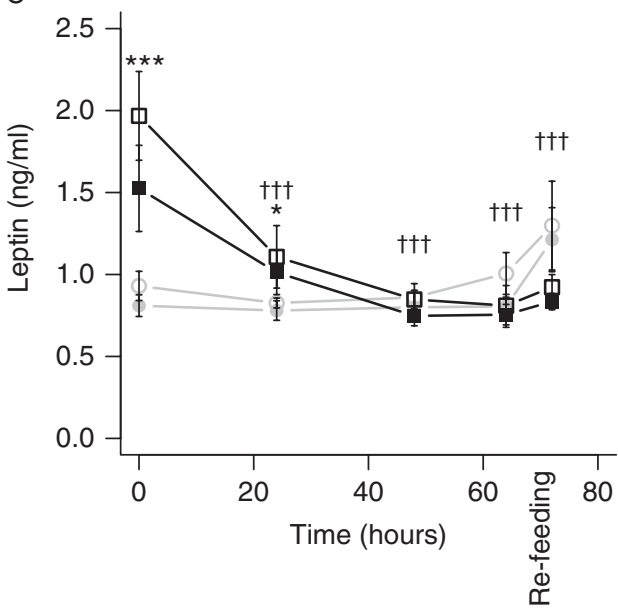

B
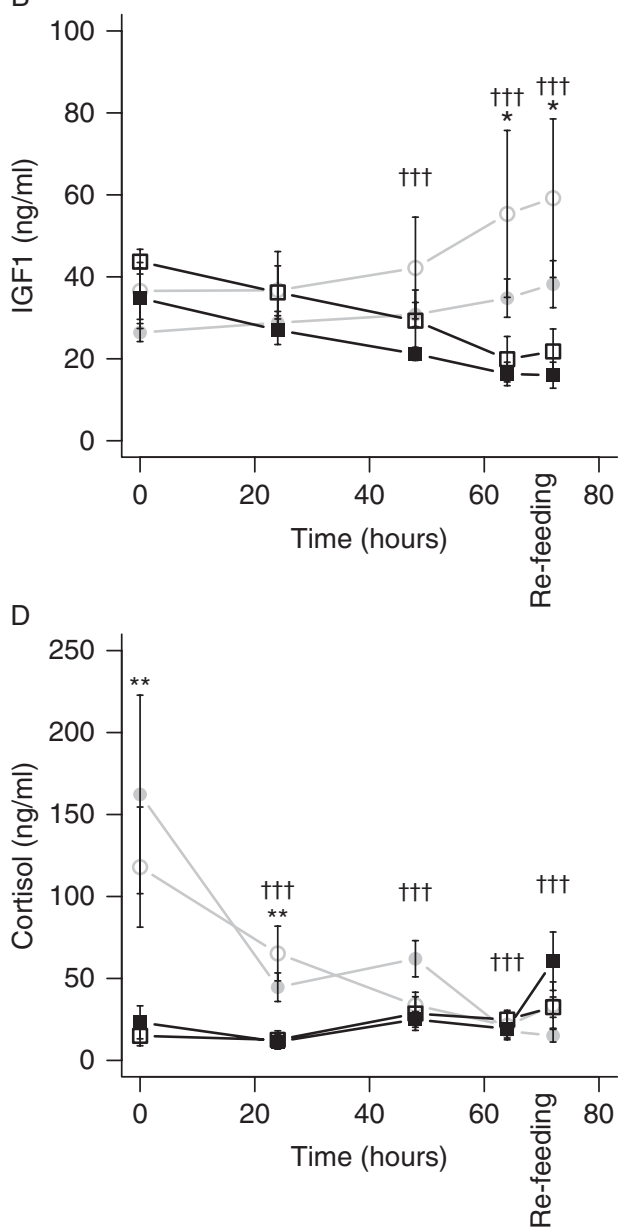

Figure 5

Hormone responses during fasting. Sheep offspring (adult females) previously exposed to either a normal (NORM) diet or $50 \%$ undernutrition (LOW) during late gestation and either a conventional (CONV) or a highcarbohydrate-high-fat (HCHF) diet in early postnatal life (0-6 months) but subsequently transferred to a normal diet (0.5-2 years) were subjected to 3 days of fasting, starting at time $=0 \mathrm{~h}$. They were all re-fed at time $=71 \mathrm{~h}$.

fasting (as marked with crosses in Fig. 5B) in contrast to those in NORM sheep, where, as expected, they decreased. Pre-fasting IGF1 concentrations did not differ between the LOW and NORM sheep. The differences in IGF1 secretion between the LOW and NORM sheep could not be related to insulin, since decreases in insulin concentrations during fasting were similar in all the treatment groups (data not shown) and were similar to the decreases observed during adolescence (Fig. 2A).

Adult LOW sheep had markedly lower basal plasma leptin concentrations (Fig. 5B). The NORM sheep exhibited a marked reduction in leptin concentrations as expected in response to fasting, which was also observed in all the adolescent lambs. On the other hand, this

(A) Insulin, (B) IGF1, (C) leptin and (D) cortisol concentrations. White box: NORM-CONV (females $n=4$ ), black box: NORM-HCHF (females $n=4)$, white circle: LOW-CONV (females $n=5$ ), and black circle: LOW-HCHF (females $n=4$ ); $* /^{\dagger} P<0.05, * * /{ }^{\dagger \dagger} P<0.01$, and $* * * /{ }^{t+\dagger} P<0.001$, where * denotes a difference in the actual concentration and $t$ the response corrected for the baseline value. The NORM offspring are shown in black and the LOW offspring in grey.

normal leptin response disappeared in the LOW sheep as they became adults (Fig. 5C), which in contrast exhibited increased leptin concentrations during fasting and after re-feeding (marked with crosses).

At baseline, the LOW sheep had higher cortisol concentrations than the NORM sheep (Fig. 5D). During fasting, cortisol concentrations decreased in the LOW sheep (marked with crosses in Fig. 5D), whereas they remained unaffected in the NORM sheep (Fig. 5D).

\section{Discussion}

The three main findings of this study were as follows: i) prenatally undernourished offspring did not exhibit

Published by Bioscientifica Ltd 
specific alterations/adaptations to starvation or reveal any metabolic or endocrine alterations to stress (adrenalin) or abundance of nutrients (propionate). However, prenatal undernutrition induced alterations in several endocrine systems exerting control (leptin) or being controlled (hypothalamic-pituitary-adrenal (HPA) axis and GHIGF1 axis) at the hypothalamic-pituitary level, but this did not become manifested until adult life. ii) A postnatal HCHF diet interfered with pancreatic $\beta$-cell secretion in ruminants. iii) The early postnatal dietary effects could be reversed by feeding a control grass-based diet from 6 months of age.

\section{Prenatally undernourished offspring did not exhibit any adaptations to stress or abundance or shortage of nutrients in early postnatal life}

We hypothesised that offspring that are undernourished during fetal life would have adapted to a stressful postnatal life characterised by food shortage. However, these animals behaved metabolically in a way similar to the prenatal control group, which argues against our hypothesis. Alternatively, the challenge tests carried out in this study might not be useful alternatives to the glucose challenge test to find individuals at a risk of developing T2D at an early time point in life. However, we cannot rule out that other metabolites or hormones would have revealed another pattern and therefore will not reject these challenge tests being used in the search for markers characterising prenatal programming. Nor can we argue that prenatally undernourished offspring are adapted to a life characterised by food shortage. However, one limitation of this study is the lack of an additional LOW-LOW control group. Thus, we cannot rule out that postnatal undernutrition could have had a beneficial effect on survival strategies, and this remains to be examined in future studies. Finally, the early separation from the dams and artificial milk feeding could have blurred some of the programming effects. However, bottle-feeding was the only option as we wanted to feed a diet with a high percentage of fat and by-pass the fore stomachs.

\section{Exposure to a high-fat diet in early postnatal life causes hyperlipidaemia and reduces pancreatic $\beta$-cell function in ruminants}

Lambs fed the HCHF diet during early neonatal life had very high plasma TG, NEFA and leptin concentrations and indications of compromised liver function in the form of increased plasma concentrations of GGT, which is a marker of non-alcoholic fatty liver disease and reduced liver function (Fraser et al. 2008, Akehi et al. 2010). The propionate challenge test was carried out during fasting in adolescent lambs to determine differences in hepatic capacity for gluconeogenesis, which is essential for the maintenance of glucose homeostasis during fasting. Propionate is one of the major substrates in gluconeogenesis in ruminants (Leng et al. 1967), and an impaired glucose response to a propionate bolus injection has been used in clinics to reveal hepatic insufficiency in ruminants (Gröhn et al. 1985). Plasma glucose concentrations thus increased as expected in response to propionate, and this was associated with a transient increase in lactate concentrations in the fasted animals. This has also been reported in a previous sheep study indicating that part of the propionate in addition to glucose will be converted to lactate as well (Cole \& Hallford 1994). The initial glucose response ( $\mathrm{AUC}_{\text {glucose }}$ ) during the first 15-30 min of the propionate tolerance test was of a similar magnitude in all the treatment groups, but in HCHF lambs the insulin response following propionate injection was substantially reduced. We previously reported that lambs exposed to the HCHF diet until adolescence also had suppressed insulin responses during an intravenous glucose tolerance test (Kongsted et al. 2013). Propionate has been shown to be an insulin secretagogue in addition to glucose (Sano et al. 1993), and it is likely that the high-fat diet, in general, reduced $\beta$-cell responsiveness to major secretagogues such as glucose and propionate. Interestingly, propionate has also been used as a diagnostic tool in ruminants to identify diabetic animals (Takasu et al. 2007), where a reduced insulin response was observed just as we had observed in the present study. The reduced $\beta$-cell responsiveness would also be an important factor explaining why the HCHF lambs even $2 \mathrm{~h}$ after injection continued to have markedly elevated glucose concentrations compared with the NORM lambs.

At first glance, it was surprising that we did not find any strong indications of reduced glucose production in the HCHF lambs after propionate injection, but we did observe a delayed glucose response pointing in that direction. In other studies where liver cirrhosis was experimentally induced, the gluconeogenic response was delayed and reduced (Gröhn 1985). The discrepancy between their study and the present study could be differences in the experimental set-ups as their sheep were non-obese sheep with presumably normal $\beta$-cell function, while our HCHF lambs clearly had severely reduced insulin-secretory responses, which would have

Published by Bioscientifica Ltd 
reduced glucose uptake in peripheral tissues, as reflected in higher $\mathrm{AUC}_{\text {glucose }}: \mathrm{AUC}_{\text {insulin }}$ ratios.

\section{Postnatal diet effects are reversible whereas prenatal impacts on endocrine function become manifested with age}

Alteration of the diet to a moderate grass-based diet for 1.5 years also reduced body weights (Nielsen et al. 2012), and most of the effects of the HCHF diet observed previously (high TG, NEFA, GTT and leptin concentrations) disappeared, indicating that influences of the postnatal diet were reversible. This is in agreement with the findings of other studies, where diet and body fat corrections have been shown to reduce hyperlipidaemia and consequently restore liver function and insulin sensitivity (Tilg \& Moschen 2010).

Surprisingly, however, we found very strong impacts of the prenatal diet on IGF1, leptin and cortisol concentrations observed in adult life. While IGF1 and leptin concentrations decreased in all the adolescent lambs (cortisol concentrations were unfortunately not measured in the young animals), and in the adult NORM sheep during fasting as expected (Cole \& Hallford 1994, Kiani et al. 2011), the response in the LOW sheep was either abolished (leptin) or the exact reverse (IGF1) of what was expected. IGF1 is one of the most important factors regulating postnatal growth. The majority of endocrine IGF1 is synthesised and released from the liver in response to the binding of $\mathrm{GH}$ to the high-affinity receptors on hepatocytes, and synthesis of these receptors is in turn stimulated by insulin (Breier et al. 1986). The present study indicates that prenatal undernutrition might cause uncoupling or disturbance of the GH-IGF1 axis during fasting, most likely distal to the level of the GH receptor in the liver, and this disturbance was not evident in young animals, but became manifested with age. Additionally, the normal fasting-induced depression of plasma leptin concentrations (Ahima et al. 1996) was abolished in the LOW sheep, as they became adults, and leptin concentrations instead tended to increase during fasting. In studies with humans and animals with a normal fetal development, it has been shown that the administration of exogenous leptin during fasting, to prevent the normal fasting-induced depression, caused dysregulation of the GH-IGF1 axis (Ahima et al. 1996, Chan et al. 2008). The hypothalamus is one of the main target organs of leptin, and upon binding to $\mathrm{OB}$ receptors in the hypothalamus, leptin can interfere with several neuroendocrine axes such as the HPA axis, the GH-IGF1 axis, the hypothalamic-pituitary-gonadal axis and hypothalamic-pituitary-thyroid axis as well as control appetite (Blüher \& Mantzoros 2004).

In addition, prenatal undernutrition apparently interfered with HPA axis function as the sheep became adults. Cortisol concentrations are expected to increase if anything during fasting, but in the LOW sheep, we observed a dramatic decrease during fasting from high levels observed during the fed state. When corrected for body weight, the LOW animals both as lambs and adults had increased adrenal weights (Nielsen et al. 2012), which together with the elevated cortisol concentrations could reflect hyperactivity of the HPA axis. A correlation between low birth weight and adult hyperactivity of the HPA axis has also been reported in human studies (Phillips et al. 1998).

We suggest that these abnormalities of fastinginduced responses in IGF1 and cortisol in the adult sheep exposed to nutrient deprivation during late fetal life potentially could be coupled to the dysregulation of the GH-IGF1 axis as well as the HPA axis by leptin at the site of the hypothalamus and/or pituitary. If this is correct, other key regulatory functions of the hypothalamus and pituitary, such as control of appetite and thyroid and reproductive functions, could potentially be programmed in response to adverse exposures during fetal life as well. To support such an assumption, we did, in fact, find strong impacts of prenatal undernutrition (LOW) on food preference (Nielsen et al. 2012) and the thyroid axis, where the LOW females became hyperthyroid (Johnsen et al. 2013). It is interesting that in this study the LOW offspring during the first few weeks of life, when they were fed ad libitum, actually preferred to ingest the (for a ruminant atypical) high-fat dairy cream over the highstarch maize, suggesting that an increased appetite for a high-fat diet can indeed be programmed during prenatal life. HPA axis activation and cortisol have been shown to enhance visceral obesity, and prenatal impacts that render the HPA axis hyperactivated during postnatal life (Anagnostis et al. 2009, Purnell et al. 2009) may thus increase the risk of visceral obesity upon exposure to a high-fat diet. This is precisely what we had observed in the LOW-HCHF lambs (Nielsen et al. 2012). Visceral obesity is one of the symptoms characterising the metabolic syndrome and has been reported to be correlated with insulin resistance and T2D (Kim et al. 2011).

We suggest that dysregulation in the coordination of hypothalamic-pituitary endocrine function and leptin may be a key factor coupling fetal undernutrition with a predisposition for developing metabolic disorders.

Published by Bioscientifica Ltd. 
In conclusion, in utero undernourished offspring did not reveal strong metabolic adaptations to an environment characterised by stress and food limitations. However, very clear effects of fetal programming of several endocrine systems exerting control (leptin) or being controlled (HPA axis and GH-IGF1 axis) at the hypothalamic-pituitary level were observed, although this was manifested only in adult sheep and not in adolescents. As alterations in these axes have been reported to be correlated to insulin resistance, (visceral) obesity and T2D, further studies are needed to explore possibilities for interventions aiming to prevent adverse outcomes later in life, specifically through targeting of these endocrine axes.

\section{Declaration of interest}

The authors declare that there is no conflict of interest that could be perceived as prejudicing the impartiality of the research reported.

\section{Funding}

This study was supported by the Danish Council for Strategic Research (grant numbers 09-059921, 09 067124).

\section{Author contribution statement}

$\mathrm{M} O \mathrm{~N}$ developed the animal model and contributed to the evaluation of data and writing of the manuscript. A $\mathrm{H} \mathrm{K}$ was in charge of the experimental planning and procedures and histological work and the main author of this manuscript. M P T, S V H and V G C participated in the management of animals, glucose and insulin measurements, practical conduct of in vivo tolerance tests and data evaluation. D B conducted the IGF1, leptin and cortisol hormone analyses and $T L$ was in charge of metabolite studies. A T conducted all the statistical analyses. B Q contributed to the experimental design and evaluation of data.

\section{Acknowledgements}

This study was part of the research programme of the Centre for Fetal Programming (CFP). The authors acknowledge D S Jensen and R Jensen for their valuable technical assistance. They also express their gratitude to the following people for their expert help with the handling of experimental animals, killing and samplings: the experimental farm staff at the Faculty of Life Sciences, University of Copenhagen, Denmark; PhD students A Kiani, W Jørgensen, T Jørgensen and A N Rasmussen; and animal science students A C F Støy, M B Petersen, H F Hedegaard, M Kjærgaard, K F Dahlbach, $M P$ Jensen, $B C$ Jensen and S R Thomasen.

\section{References}

Ahima RS, Prabakaran D, Mantzoros C, Qu D, Lowell B, Maratos-Flier E \& Flier JS 1996 Role of leptin in the neuroendocrine response to fasting. Nature 382 250-252. (doi:10.1038/382250a0)

Akehi Y, Tsutsumi Y, Tatsumoto A, Yoshida R, Ohkubo K, Takenoshita H, Kudo T, Ashida K, Anzai K, Yamashita T et al. 2010 Serum $\gamma$-glutamyltransferase, triglyceride and total cholesterol are possible prediabetic risk markers in young Japanese men. Endocrine Journal 57 981-989. (doi:10.1507/endocrj.K10E-174)

Anagnostis P, Athyros VG, Tziomalos K, Karagiannis A \& Mikhailidis DP 2009 The pathogenetic role of cortisol in the metabolic syndrome: a hypothesis. Journal of Clinical Endocrinology and Metabolism 94 2692-2701. (doi:10.1210/jc.2009-0370)

Blüher S \& Mantzoros CS 2004 The role of leptin in regulating neuroendocrine function in humans. Journal of Nutrition $\mathbf{1 3 4}$ 2469S-2474S

Breier BH, Bass JJ, Butler JH \& Gluckman PD 1986 The somatotrophic axis in young steers: influence of nutritional status on pulsatile release of growth hormone and circulating concentrations of insulin-like growth factor 1. Journal of Endocrinology 111 209-215. (doi:10.1677/joe.0. 1110209)

Chan JL, Williams CJ, Raciti P, Blakeman J, Kelesidis T, Kelesidis I, Johnson ML, Thorner MO \& Mantzoros CS 2008 Leptin does not mediate short-term fasting-induced changes in growth hormone pulsatility but increases IGF-I in leptin deficiency states. Journal of Clinical Endocrinology and Metabolism 93 2819-2827. (doi:10.1210/ jc.2008-0056)

Cleal JK, Poore KR, Boullin JP, Khan O, Chau R, Hambidge O, Torrens C, Newman JP, Poston L, Noakes DE et al. 2007 Mismatched pre- and postnatal nutrition leads to cardiovascular dysfunction and altered renal function in adulthood. PNAS 104 9529-9533. (doi:10.1073/pnas. 0610373104)

Cole NA \& Hallford DM 1994 Influence of a propionate load in fed or unfed lambs on blood metabolites and hormone patterns. Journal of Animal Science 72 2141-2148.

Fall CHD, Sachdev HS, Osmond C, Lakshmy R, Biswas SD, Prabhakaran D, Tandon N, Ramji S, Reddy KS, Barker DJP et al. 2008 Adult metabolic syndrome and impaired glucose tolerance are associated with different patterns of BMI gain during infancy: data from the New Delhi birth cohort. Diabetes Care 31 2349-2356. (doi:10.2337/dc08-0911)

Forsen T, Eriksson J, Tuomilehto J, Reunanen A, Osmond C \& Barker D 2000 The fetal and childhood growth of persons who develop type 2 diabetes. Annals of Internal Medicine 133 176-182. (doi:10.7326/00034819-133-3-200008010-00008)

Fraser A, Ebrahim S, Davey Smith G \& Lawlor DA 2008 The associations between birthweight and adult markers of liver damage and function. Paediatric and Perinatal Epidemiology 22 12-21.

Gavete ML, Martín MA, Alvarez C \& Escrivá F 2005 Maternal food restriction enhances insulin-induced GLUT-4 translocation and insulin signaling pathway in skeletal muscle from suckling rats. Endocrinology 146 3368-3378. (doi:10.1210/en.2004-1658)

Gluckman PD, Hanson MA \& Pinal C 2005 The developmental origins of adult disease. Maternal \& Child Nutrition 1 130-141. (doi:10.1111/ j.1740-8709.2005.00020.x)

Gröhn Y 1985 Propionate loading test for liver function in spontaneously ketotic dairy cows. Research in Veterinary Science 39 24-28.

Gröhn Y, Bruss ML \& Lindberg LA 1985 Propionate loading test for liver function during experimental liver necrosis in sheep. American Journal of Veterinary Research 46 952-958.

Husted SM, Nielsen MO, Tygesen MP, Kiani A, Blache D \& Ingvartsen KL 2007 Programming of intermediate metabolism in young lambs affected by late gestational maternal undernourishment. American Journal of Physiology. Endocrinology and Metabolism 293 E548-E557. (doi:10.1152/ajpendo.00441.2006)

Johnsen L, Kongsted AH \& Nielsen MO 2013 Prenatal undernutrition and postnatal overnutrition alter thyroid hormone axis function in sheep. Journal of Endocrinology 216 389-402. (doi:10.1530/JOE-12-0389)

Kiani A, Nielsen MO, Tauson AH, Tygesen MP, Husted SM \& Chwalibog A 2011 Long-term effects of foetal undernutrition on intermediary metabolism in growing lambs. Archives of Animal Nutrition 65 46-54. (doi:10.1080/1745039X.2010.533551) 
Kim LJ, Nalls MA, Eiriksdottir G, Sigurdsson S, Launer LJ, Koster A, Chaves PHM, Jonsdottir B, Garcia M, Gudnason V et al. 2011 Associations of visceral and liver fat with the metabolic syndrome across the spectrum of obesity: the AGES-Reykjavik study. Obesity 19 1265-1271. (doi:10.1038/oby.2010.291)

Kongsted AH, Tygesen MP, Husted SV, Oliver MH, Tolver A, Christensen VG, Nielsen JH \& Nielsen MO 2013 Programming of glucose-insulin homoeostasis: long-term consequences of pre-natal versus early post-natal nutrition insults. Evidence from a sheep model. Acta Physiologica [in press]. (doi:10.1111/apha.12080)

Leng RA, Steel JW \& Luick JR 1967 Contribution of propionate to glucose synthesis in sheep. Biochemical Journal 103 785-790.

Misra A \& Vikram NK 2004 Insulin resistance syndrome (metabolic syndrome) and obesity in Asian Indians: evidence and implications. Nutrition 20 482-491. (doi:10.1016/j.nut.2004.01.020)

Nielsen MO, Kongsted AH, Thygesen MP, Strathe AB, Caddy S, Quistorff B, Jórgensen W, Christensen VG, Husted S, Chwalibog A et al. 2012 Late gestation undernutrition can predispose for visceral adiposity by altering fat distribution patterns and increasing the preference for a high-fat diet in early postnatal life. British Journal of Nutrition 109 2098-2110. (doi:10.1017/S0007114512004199)

Petry CJ, Dorling MW, Pawlak DB, Ozanne SE \& Hales CN 2001 Diabetes in old male offspring of rat dams fed a reduced protein diet. International Journal of Experimental Diabetes Research 2 139-143. (doi:10.1155/EDR. 2001.139)

Phillips DIW, Barker DJP, Fall CHD, Seckl JR, Whorwood CB, Wood PJ \& Walker BR 1998 Elevated plasma cortisol concentrations: a link between low birth weight and the insulin resistance syndrome? Journal of Clinical Endocrinology and Metabolism 83 757-760. (doi:10.1210/jc.83.3.757)

Purnell JQ, Kahn SE, Samuels MH, Brandon D, Loriaux DL \& Brunzell JD 2009 Enhanced cortisol production rates, free cortisol, and
$11 \beta$-HSD-1 expression correlate with visceral fat and insulin resistance in men: effect of weight loss. American Journal of Physiology. Endocrinology and Metabolism 296 E351-E357. (doi:10.1152/ajpendo. 90769.2008)

R Development Core Team. A language and environment for statistical computing. 2009. R Foundation for Statistical Computing.

Sano H, Hattori N, Todome Y, Tsuruoka J, Takahashi H \& Terashima Y 1993 Plasma insulin and glucagon responses to intravenous infusion of propionate and their autonomic control in sheep. Journal of Animal Science 71 3414-3422.

Spalding A, Kernan J \& Lockette W 2009 The metabolic syndrome: a modern plague spread by modern technology. Journal of Clinical Hypertension 11 755-760. (doi:10.1111/j.1751-7176.2009.00191.x)

Stettler N, Kumanyika SK, Katz SH, Zemel BS \& Stallings VA 2003 Rapid weight gain during infancy and obesity in young adulthood in a cohort of African Americans. American Journal of Clinical Nutrition $\mathbf{7 7}$ 1374-1378.

Stettler N, Stallings VA, Troxel AB, Zhao J, Schinnar R, Nelson SE, Ziegler EE \& Strom BL 2005 Weight gain in the first week of life and overweight in adulthood. Circulation 111 1897-1903. (doi:10.1161/01.CIR. 0000161797.67671.A7)

Takasu M, Ohba Y, Iguchi T, Nishii N, Maeda S \& Kitagawa H 2007 Propionate tolerance test for determination of insulin secretion in a hyperglycemic Japanese Black steer. Journal of Veterinary Medical Science 69 985-987. (doi:10.1292/jvms.69.985)

Tilg H \& Moschen A 2010 Weight loss: cornerstone in the treatment of non-alcoholic fatty liver disease. Minerva Gastroenterologica e Dietologica 56 159-167.

Vickers MH, Breier BH, Cutfield WS, Hofman PL \& Gluckman PD 2000 Fetal origins of hyperphagia, obesity, and hypertension and postnatal amplification by hypercaloric nutrition. American Journal of Physiology. Endocrinology and Metabolism 279 E83-E87.

Received in final form 13 August 2013

Accepted 19 August 2013

Accepted Preprint published online 19 August 2013
C 2013 Society for Endocrinology Printed in Great Britain 